\title{
A pilot trial of quetiapine, lithium, or placebo added to divalproex sodium for hypomanic or manic episodes in ambulatory adults with bipolar I disorder
}

Victoria E. Cosgrove ${ }^{1,2^{*}}$ DD, Santiago Allende ${ }^{2}$, lola Gwizdowski ${ }^{1 \wedge}$, E. Grace Fischer ${ }^{1}$, Michael Ostacher ${ }^{1,2}$ and Trisha Suppes ${ }^{1,2}$

\begin{abstract}
Background: Many patients with bipolar I disorder do not respond to monotherapy treatment with mood-stabilizing medications, and combination regimens are commonly used in both inpatient and outpatient settings for the acute and maintenance treatment of bipolar disorder. We studied whether combination therapy is more effective than monotherapy for the acute treatment of subjects with bipolar I disorder currently experiencing manic symptoms. The primary hypothesis was that combination treatments would be associated with greater reductions in symptoms of mania and hypomania than monotherapy alone. The secondary hypothesis was that combination therapies would be associated with lower depression levels than monotherapy alone. Last, a post-hoc exploratory aim was used to examine whether the effect of side effect severity on risk-of-dropout would be greater in combination therapies than in monotherapy alone.
\end{abstract}

Results: In this 12-week, double-blind, placebo-controlled ambulatory pilot trial, participants $(n=75)$ with bipolar I disorder were randomly assigned to: (1) monotherapy divalproex plus placebo (DVP + PBO), (2) combination therapy of divalproex plus blinded lithium (DVP + Li) or (3) divalproex plus blinded quetiapine (DVP + QTP). Combination therapies (vs. monotherapy) were not associated with improved symptoms of mania, hypomania or depression. The effect of side effect severity on study retention did not differ between combination therapies and monotherapy. However, the risk-of-dropout was significantly greater in the DVP + Li arm versus the DVP + PBO arm.

Conclusions: No longitudinal differences in mania, hypomania or depression were found between combination therapies and monotherapy. The effect of side effect severity on study retention did not differ between groups. Due to the small sample size and differential rates of attrition between treatment arms, results of this pilot trial must be interpreted with caution.

Trial registration ClinicalTrials.gov identifier: NCT00183443

Keywords: Monotherapy divalproex, Divalproex plus blinded lithium, Divalproex plus blinded quetiapine

*Correspondence: veileen@stanford.edu

Iola Gwizdowski-deceased

2 Department of Psychiatry and Behavioral Sciences, Stanford University

School of Medicine, 401 Quarry Road, Stanford, CA 94305, USA

Full list of author information is available at the end of the article

\section{Background}

Bipolar disorder is a chronic mental illness often requiring lifelong treatment (Suppes et al. 1991). Effectively treating bipolar disorder is often challenging, in part because of the disorder's chronic and recurrent nature. Most treatment guidelines recommend monotherapy as 
a first-line treatment option, reserving treatment with multiple medications as an option for more severely ill or non-responsive patients. Yet, combination therapies are commonly used in both inpatient and outpatient settings as initial treatment (Yatham et al. 2018; Grande and Vieta 2015).

Adverse events are one major consequence of polypharmacy in bipolar I disorder, leading to diminished quality of life and global functioning, poorer medication adherence, and higher rates of treatment dropout (Brooks et al. 2010; Gaudiano et al. 2008; Scott 2002). Both patient nonadherence to psychotropic medication regimens and early dropout further complicate treatment, possibly explaining an efficacy-effectiveness gap observed in bipolar disorder research in which clinical trials report higher efficacy rates than those observed in clinical practice (Gaudiano et al. 2008; Scott 2002).

Patients with bipolar I disorder do not exclusively have episodes of mania or depression; they may also experience episodes of hypomania, and hypo/mania and depression with mixed features. In spite of this, the evidence base for the treatment of hypomanic episodes in bipolar I disorder is very limited, and to date there are no established treatments for hypomanic episodes. Little attention has been directed at these types of episodes as a treatment target, though it is recognized that hypomania itself can signal destabilization in patients with bipolar I disorder.

A growing body of evidence suggests negligible overall improved outcomes for combination therapies, indicating that the question of monotherapy versus combination therapy remains highly relevant (Nierenberg et al. 2013; Altshuler et al. 2017; Geddes et al. 2011). In clinical practice, divalproex is commonly combined with either quetiapine or lithium to treat hypomania or mania. The present pilot study is a 12-week acute phase, doubleblind, placebo-controlled trial of monotherapy versus combination medication comparing open-label divalproex plus adjunctive blinded slow-release lithium carbonate, blinded quetiapine, or placebo for the treatment of hypomanic or manic symptoms during hypomanic, manic, or mixed episodes in outpatient participants with bipolar I disorder.

\section{Methods}

This is a randomized, double-blind, placebo-controlled, 12-week pilot investigation of the safety and effectiveness of monotherapy divalproex plus placebo (DVP $+\mathrm{PBO}$ ) versus combinations of divalproex plus blinded lithium $(\mathrm{DVP}+\mathrm{Li})$ or divalproex plus blinded quetiapine (DVP + QTP) for treatment of hypomania or mania in outpatients with bipolar I disorder.

\section{Procedures}

This study was approved by Institutional Review Boards of the University of Texas Southwestern Medical Center and the Stanford University School of Medicine, written informed consent was obtained, and the study was registered at ClinicalTrials.gov (identifier: NCT00183443).

Outpatient participants diagnosed with bipolar I disorder as defined in DSM-IV between the ages of 18-65 and experiencing hypomania or mania with a Young Mania Rating Scale (YMRS) score of $\geq 15$ were eligible for study entry (see Table 1 for inclusion/exclusion criteria). Although patients were excluded for current use of DVP $+\mathrm{Li}$, patients were not excluded for current monotherapy use of DVP, Li or QTP without a history of intolerance, toxicity or adverse experiences. Once

Table 1 Inclusion and exclusion criteria

\begin{tabular}{|c|c|}
\hline Inclusion criteria & Exclusion criteria \\
\hline $\begin{array}{l}\text { - English-speaking adults at least } 18 \text { years old } \\
\text { - History of DSM-IV bipolar I disorder, confirmed by Structured Clinical } \\
\text { Interview } \\
\text { - Experiencing hypomania or mania with a score on Young Mania Rating } \\
\text { Scale } \geq 15 \text { and meeting DSM-IV criteria for a current hypomanic, manic, or } \\
\text { mixed episode } \\
\text { - Desire to seek treatment for bipolar I disorder } \\
\text { - Written informed consent obtained and willingness to perform study } \\
\text { procedures confirmed } \\
\text { - Agree to taper existing ineffective medications and be randomized to } \\
\text { one of the three study conditions } \\
\text { - No use of antidepressants for } 1 \text { month prior to randomization (3 months } \\
\text { for fluoxetine) }\end{array}$ & $\begin{array}{l}\text { - Patients who are euthymic (well) on their current medications } \\
\text { - Patients who are already taking combination DVP + Li or DVP + QTP } \\
\text { - Patients with a history of partial or nonresponse to DVP, Li, QTP, or any } \\
\text { combination of those medications, documented by serum levels } \\
\text { - History of intolerance or toxicity to DVP, Li, or QTP } \\
\text { - History of adverse experiences to DVP, Li, or QTP } \\
\text { - Disorders that would contraindicate or limit the use of Li, including derma- } \\
\text { tological conditions, such as psoriasis or acne vulgaris, or kidney failure } \\
\text { - Disorders that would contraindicate the use of DVP, including clinically } \\
\text { significant active hepatitis or hepatic failure as evidenced by abnormal } \\
\text { laboratory values } \\
\text { - Impaired cardiac function as evidenced by positive EKG in subjects age } 50 \\
\text { or over } \\
\text { - Patients with unstable medical illnesses within the past } 2 \text { months } \\
\text { - Current suicidal ideation or intent } \\
\text { - Substance abuse or dependency within the past month } \\
\text { - Pregnant (i.e., positive urine pregnancy test) or nursing women or plan- } \\
\text { ning to conceive }\end{array}$ \\
\hline
\end{tabular}


enrolled, participants were randomized to one of three treatment conditions: DVP $+\mathrm{PBO}$ (monotherapy), $\mathrm{DVP}+\mathrm{Li}$, or DVP + QTP. Participants were evaluated weekly for the first 4 weeks then at 2-week intervals until the completion of the 12-week study. Participants, study physicians, and raters were blind to group assignment.

At screening, a diagnosis of bipolar I disorder (BDI) was confirmed with the Structured Clinical Interview for DSM-IV (SCID) (First et al. 1996). The screening visit also consisted of confirmation of inclusion and exclusion criteria, laboratory work, a physical exam including an eye exam, and an EKG (if subject was $>50$ years old). Participants prescribed other psychiatric medications at study entry were simultaneously started on study medication and titrated off prior medications at a rate of $33 \%$ every 3-4 days to assure that the taper was complete by week 2 (Faedda et al. 1993). Randomization occurred if screening visit results confirmed eligibility. Efron's biased coin design, with stratified randomization, separately for participants who reported rapid cycling in the preceding 12 months and those who did not, was used to ensure even distribution across treatment arms (Efron 1971).

At each study visit, study staff administered clinical assessment measures. The primary outcome measure was change in YMRS scores (Young et al. 1978), an 11-item clinician-rated measure providing data on degree and severity of manic symptoms. The Hamilton Rating Scale for Depression (HAM-D) (Hamilton 1960; Hamilton 1967; Williams 1988), a 17-item measure of symptom severity for depression, served as a secondary outcome measure. In addition to weight change, a total of 31 side effects were rated on a 4-point Likert-type scale ranging from 1 (absent) and 2 (mild) to 3 (moderate) and 4 (severe).

\section{Blinded medication adjustments}

A study psychiatrist blind to treatment assignment adjusted study medications based on clinical symptoms at each study visit. Open-label divalproex was titrated to a stable dose with periodic serum level checks on blood levels to confirm levels were within the therapeutic window (see below). Blinded medications were dosed at one capsule/day for the first 2 days, two capsules/day for the second 2 days, three capsules/day for the next 3 days, followed by four capsules/day for the remainder of the trial. Study medication was increased if a subject had no change or worsened, as evaluated by the CGI-BP and the YMRS. Same day oversight of all adjustments was by an unblinded psychiatrist to assure that doses and levels were in the prespecified range; the unblinded psychiatrist would substitute placebo if a dosage increase ordered by the blinded psychiatrist was outside the dosage range.

\section{Dosing and serum levels of DVP}

DVP was titrated as $250 \mathrm{mg} /$ day $\times 2$ days; $500 \mathrm{mg} /$ day $\times 2$ days; $750 \mathrm{mg} /$ day until the next visit, at which time a serum blood level should be drawn. The approximate target dose range was $750-2000 \mathrm{mg} /$ day, which can be administered in one dose. All subjects were maintained at a minimum level of DVP of $50 \mathrm{mg} / \mathrm{L}$ and a maximum of $125 \mathrm{mg} / \mathrm{L}$. Patients unable to tolerate these minimums, despite decrease of blinded medication to minimum dosage/serum levels, were discontinued. Once this minimum of DVP was reached, all other medication adjustments took place only in the blinded medication (QTP, Li, or PBO).

\section{Dosing and serum levels of lithium}

The target goal for $\mathrm{Li}$ was $0.8 \mathrm{mEq} / \mathrm{L}$ or greater, and the minimum was $0.6 \mathrm{mEq} / \mathrm{L}$. Lithium was started at $300 \mathrm{mg} /$ day, and gradually increased to $900 \mathrm{mg}$ /day over the course of a week (Table 3). Lithium level was drawn within 5 days after reaching minimum initial dosing of $900 \mathrm{mg}$ /day (week 2), and at weeks 4, 6, 12, 20, and 26 . Serum levels were targeted to 0.6 to $<1.2 \mathrm{mEg} / \mathrm{L}$.

\section{Dosing of quetiapine}

QTP was initiated at $50 \mathrm{mg}$, increased to $100 \mathrm{mg}$, then by $100 \mathrm{mg} /$ day until $400 \mathrm{mg}$ was reached by Day 5 with a goal of $600 \mathrm{mg}$ during week 2 . In order to maintain with real world applicability, these targets were encouraged by an unblinded physician, but decreases or slower titration was allowed for tolerability. A minimum of $100 \mathrm{mg} /$ day of QTP was required to remain in the study.

\section{Discontinuation and use of other medications}

Subjects were terminated from the protocol if the CGI$\mathrm{BP}$ rating for change from the previous measurement was "much worse" or "very much worse" if confirmed at a return clinic visit. Subjects who were unable to maintain minimum doses or levels of any study medications (valproate serum level of $50 \mathrm{mg} / \mathrm{mL}$, lithium level of 0.6 $\mathrm{eEq} / \mathrm{L}$, or quetiapine dose of $100 \mathrm{mg}$ ) due to side effects were discontinued from the study.

Short-term use of lorazepam up to $2 \mathrm{mg} /$ day for a maximum of 5 consecutive days on no more than three occasions over the trial was permitted. No other adjunctive medication was allowed. For subjects who met response criteria at week $12\left(\right.$ YMRS $<8$, HAM- $\left.\mathrm{D}_{17}<10\right)$, a 14-week continuation period was offered. Results from this continuation trial are not reported here. 


\section{Statistical analysis}

An a priori power analysis indicated that 35 participants per arm were required to obtain $80 \%$ power for detecting an effect size of 0.6, using a one-tailed test with an alpha level of 0.05. Due to dependency in the data, an intent-to-treat linear mixed modeling approach was used to examine the primary and secondary outcome measures, change in YMRS score and change in HAM-D score, respectively (Raudenbush and Bryk 2002). Linear mixed models use listwise deletion of individual observations rather than entire persons and maximum likelihood estimation to robustly handle data that are missing at random or completely at random (Snijders and Bosker 1999). Prior to all analyses, all continuous variables, with the exception of time, were centered at their arithmetic means.

A likelihood ratio test demonstrated that the best fitting model for mania and depression, fit separately, was a random linear time model. Both models were estimated using restricted maximum likelihood and included fixed effects for treatment condition (DVP + PBO vs. DVP + Li \& DVP + PBO vs. DVP + QTP), time, their interaction and site. We did not include a 3-way interaction with site due to only six participants completing the trial at the Palo Alto VA. Separate linear mixed models were estimated to assess longitudinal differences in the monotherapy versus aggregated combination therapy groups $(\mathrm{DVP}+\mathrm{PBO}$ vs. DVP + Li \& DVP + QTP). A standardized coefficient for significant variables was derived by dividing the product of the coefficient and the standard deviation of the variable by the standard deviation of the outcome variable (Snijders and Bosker 1999). Diagnostic plots for level 1 and for level 2 did not reveal notable departures from normality, linearity, independence or constant variance.

Due to the small sample size and high rate of attrition in the present pilot study, in lieu of examining changes in the Clinical Global Impression Scale for Bipolar Disorder (CGI-BD), the Global Assessment of Functioning and the Social and Occupational Functioning Assessment Scale (SOFAS) as secondary outcome measures, a posthoc exploratory analysis was used to investigate whether attrition differed among treatment arms as a function of side effect severity. In a Cox proportional hazards model, an interaction term between treatment group and side effect severity as a time-dependent covariate was used to examine whether the effect of treatment group on timein-study depended on side effect severity. The side effect severity variable was derived by first recentering the scale to range from 0 to 3 (absent to severe) and then summing the side effect ratings for each participant. In a separate model, we tested an interaction between treatment group and number of side effects reported to compare with the side effect severity variable. Models were adjusted for YMRS and HAM-D scores.

A modified intention-to-treat analysis was performed, where only participants with at least one post-randomization follow-up visit were included in the Cox regression model. An examination of Schoenfeld residuals did not reveal violations of the proportional hazards assumption. Further, due to six of the participants completing the trial at the Palo Alto VA (vs. UT Southwestern Medical School), we estimated models with and without adjusting for the effect of site. Although a 3-way interaction would have been preferred, the unbalanced data would have rendered estimates less reliable. Last, separate models were estimated for monotherapy versus individual combination therapy (DVP + PBO vs. DVP + Li \& $\mathrm{DVP}+\mathrm{PBO}$ vs. DVP + QTP) and monotherapy versus aggregated combination therapy groups (DVP + $\mathrm{PBO}$ vs. DVP + Li \& DVP + QTP).

All analyses were conducted with the $\mathrm{R}$ statistical computing language within the RStudio IDE (Winder et al. 2019; Team R 2020). Data cleaning and structuring were completed with the tidyverse set of packages, while the linear mixed models were estimated with the nlme package (Wickham 2017; Pinheiro et al. 2013). The sjPlot package was used to generate the linear mixed model table (Lüdecke 2018). The survival and survminer packages were used to estimate the survival functions (Therneau 2020; Kassambara 2018).

\section{Results}

Of the 75 participants who were included in the study (mean $[S D]$ age $=35.5$ [10.4] years; $50.7 \%$ female), 24 were randomized to the $\mathrm{DVP}+\mathrm{PBO}$ arm (mean $[S D]$ age $=38.8$ [11.9] years; $41.7 \%$ female), 25 were randomized to DVP + Li (mean [SD] age $=33.3$ [9.1] years; $48.0 \%$ female) and 26 were randomized to DVP + QTP (mean [SD] age $=34.5$ [9.7] years; $61.5 \%$ female; see Fig. 1). As shown in Table 2, no differences in demographic or medical variables were found across the three groups. One subject was on divalproex at study start; otherwise all subjects were started on divalproex at baseline. The average number of reported side effects of at least moderate intensity in the entire cohort and per arm is shown in Table 3. Last, negligible differences in linear mixed model estimates were found between the individual and aggregated combination therapy models (see Table 4).

\section{Longitudinal analyses \\ Young Mania Rating Scale}

Results failed to demonstrate significant longitudinal differences in YMRS scores between the $\mathrm{PBO}$ and $\mathrm{Li}$ 


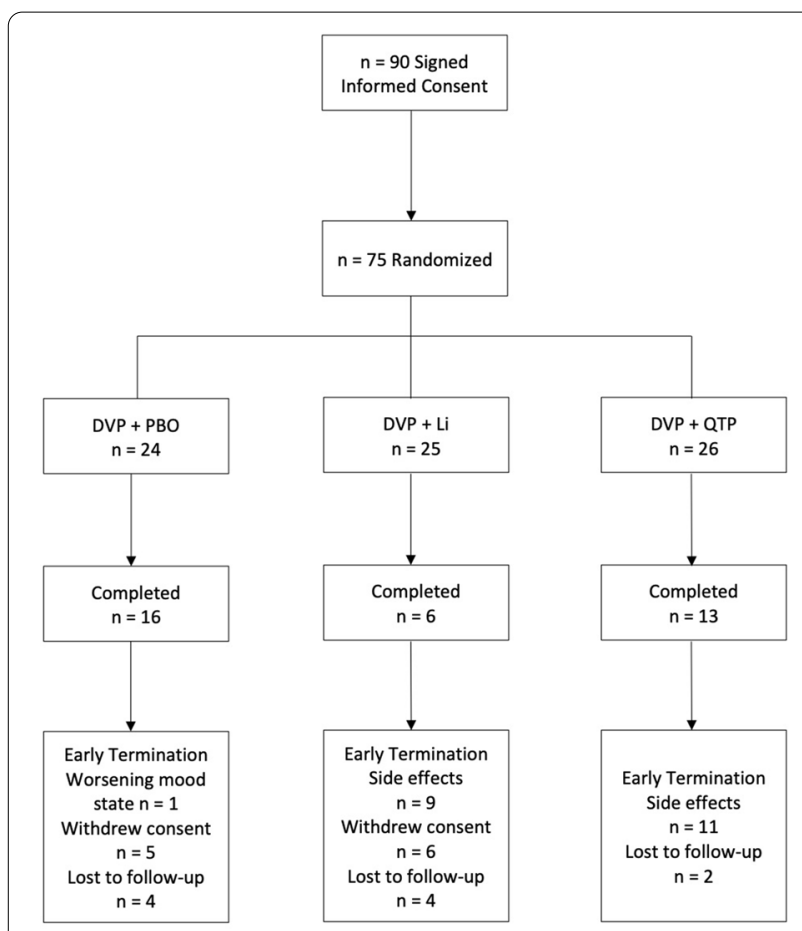

Fig. 1 Consort diagram versus QTP and side effect severity (see Table 6). Similarly, a separate model failed to find a significant interaction between $\mathrm{PBO}$ versus $\mathrm{Li}$ and number of side effects reported, $(b=-0.05$, Wald $=-0.18, p=0.859)$ as well as $\mathrm{PBO}$ versus QTP and number of side effects reported $(b=-0.00$, Wald $=-0.00, p=0.998)$.

The Cox regression estimated that the risk-of-dropout for a participant with an average side effect severity score was 3.04 times higher for the Li group than for the PBO group (see Table 6), albeit with a large confidence interval $(95 \% \mathrm{CI}=1.08-8.57)$. The simple effect for the risk-of-dropout between the PBO and QTP groups was not significant (see Table 6 and Fig. 3). There were also no significant effects of YMRS and HAM-D or Site (see Table 6). Importantly, significance remained unchanged when we excluded the six participants who were recruited to the Palo Alto VA. Moreover, estimates were negligibly different across models (see Table 6). In the monotherapy and aggregated combination therapy model, the estimated risk-of-dropout was 2.56 times higher for the combination therapy arms than for the monotherapy arm (see Table 7). Although significance for HAM-D changed when excluding Palo Alto VA participants, the Hazard ratio was negligibly different (1.06 vs. 1.07) (see Table 7).

\section{Discussion}

The primary hypothesis of this pilot trial was whether combination therapies (DVP + Li and DVP + QTP) would be associated with greater reduction in symptoms of hypomania and mania as measured by the YMRS than monotherapy alone (DVP $+\mathrm{PBO})$, while the secondary hypothesis of this exploratory trial was whether combination therapies would be associated with lower depression levels as measured by the HAM-D than those on monotherapy. In lieu of the other original secondary measures of the present pilot study, a post-hoc exploratory aim was included to examine whether the effect of side effect severity on study retention differed among combination therapies and monotherapy $(\mathrm{DVP}+\mathrm{PBO})$ during the 12-week acute phase. Given the small sample size and high rates of attrition, the results of this pilot trial must be interpreted with caution and considered as exploratory. Contrary to the primary and secondary hypotheses, results failed to demonstrate significant longitudinal differences in mania and depression scores between combination therapies and monotherapy. Moreover, the effect of side effect severity on study retention did not vary as a function of monotherapy versus combination therapies. However, the risk-of-dropout was significantly greater in the DVP $+\mathrm{Li}$ arm versus the $\mathrm{DVP}+\mathrm{PBO}$ arm.

Our provisional and exploratory null results for monotherapy versus combination therapies on symptoms of bipolar I disorder are consistent with prior literature for studies in ambulatory versus inpatient samples. For 
Table 2 Baseline characteristics of sample

\begin{tabular}{|c|c|c|c|c|c|}
\hline & $\begin{array}{l}\text { Entire cohort } \\
N=75\end{array}$ & $\begin{array}{l}\text { DVP+PBO } \\
n=24\end{array}$ & $\begin{array}{l}\text { DVP }+\mathrm{Li} \\
\mathrm{n}=25\end{array}$ & $\begin{array}{l}\text { DVP + QTP } \\
n=26\end{array}$ & Significance test \\
\hline Age at baseline (mean, SD) & $35.5(10.4)$ & $38.8(11.9)$ & $33.3(9.1)$ & $34.5(9.7)$ & $F(2)=1.91, p=0.155$ \\
\hline Women & $38(50.7 \%)$ & $10(41.7 \%)$ & $12(48.0 \%)$ & $16(61.5 \%)$ & $x^{2}(2)=2.08, p=0.354$ \\
\hline \multicolumn{6}{|l|}{ Race/ethnicity } \\
\hline Caucasian & $53(70.7 \%)$ & $18(75.0 \%)$ & $17(68.0 \%)$ & $18(69.2 \%)$ & \multirow[t]{2}{*}{$x^{2}(2)=0.33, p=0.848$} \\
\hline Other & $22(29.3 \%)$ & $6(25.0 \%)$ & $8(32.0 \%)$ & $8(30.8 \%)$ & \\
\hline YMRS score at baseline (mean, SD) & $22.97(6.20)$ & $22.92(5.72)$ & $23.84(7.49)$ & $22.19(5.32)$ & $F(2)=0.4449, p=0.643$ \\
\hline Mean HAM-D score at baseline (mean, SD) & $13.57(6.50)$ & $13.88(6.02)$ & $13.20(6.81)$ & $13.65(6.86)$ & $F(2)=0.0673, p=0.935$ \\
\hline \multicolumn{6}{|l|}{ Marital status } \\
\hline Married or cohabitating & $25(33.3 \%)$ & $7(29.2 \%)$ & $8(32.0 \%)$ & $10(38.5 \%)$ & \multirow[t]{2}{*}{$x^{2}(2)=0.52, p=0.773$} \\
\hline Single, divorced, widowed or separated & $50(66.7 \%)$ & $17(70.8 \%)$ & $17(68.0 \%)$ & $16(61.5 \%)$ & \\
\hline \multicolumn{6}{|l|}{ Education } \\
\hline High school diploma or less & $19(25.3 \%)$ & $8(33.3 \%)$ & $5(20.0 \%)$ & $6(23.1 \%)$ & \multirow[t]{3}{*}{$X^{2}(4)=2.91, p=0.573$} \\
\hline Some college or vocational & $33(44.0 \%)$ & $8(33.3 \%)$ & $11(44.0 \%)$ & $14(53.8 \%)$ & \\
\hline Associates degree or more & $23(30.7 \%)$ & $8(33.3 \%)$ & $9(36.0 \%)$ & $6(23.1 \%)$ & \\
\hline \multicolumn{6}{|l|}{ Employment status } \\
\hline Self, PT, or FT employment & $47(62.7 \%)$ & $17(70.8 \%)$ & $14(56.0 \%)$ & $16(61.5 \%)$ & \multirow[t]{2}{*}{$x^{2}(2)=1.17, p=0.556$} \\
\hline Not employed & $28(37.3 \%)$ & $7(29.2 \%)$ & $11(44.0 \%)$ & $10(38.5 \%)$ & \\
\hline Age at onset of first symptoms of depression & $12.5(6.9)$ & $12.4(8.0)$ & $13.2(6.4)$ & $11.8(6.3)$ & $F(2)=12.99, p=0.763$ \\
\hline Age at onset of first symptoms of mania & $15.2(12.6)$ & $13.4(10.3)$ & $17.4(18.4)$ & $14.6(5.6)$ & $F(2)=0.65, p=0.524$ \\
\hline Mean number of lifetime episodes of depression & $3.5(1.1)$ & $3.4(1.0)$ & $3.7(0.9)$ & $3.5(1.3)$ & $F(2)=0.50, p=0.607$ \\
\hline Mean number of lifetime episodes of mania & $3.9(0.6)$ & $3.9(0.7)$ & $4(0.0)$ & $3.8(0.9)$ & $F(2)=0.39, p=0.679$ \\
\hline Mean number of lifetime episodes of hypomania & $4.0(0.5)$ & $4.1(0.4)$ & $3.9(0.6)$ & $4.0(0.6)$ & $F(2)=1.33, p=0.272$ \\
\hline Age of diagnosis of any mood disorder & $26.7(11.2)$ & $29.5(11.2)$ & $25.4(10.2)$ & $25.5(11.9)$ & $F(2)=1.09, p=0.342$ \\
\hline Number of lifetime psychiatric hospitalizations & $1.2(3.7)$ & $1.7(6.1)$ & $1.0(1.7)$ & $1.0(1.8)$ & $F(2)=0.32, p=0.725$ \\
\hline Self-reported lifetime history of rapid cycling & $60(85.1 \%)$ & $18(75.0 \%)$ & $23(92.0 \%)$ & $22(88.0 \%)$ & $x^{2}(2)=0.40, p=0.818$ \\
\hline Self-reported lifetime history of psychosis & $26(35.1 \%)$ & $8(33.3 \%)$ & $10(40 \%)$ & $8(32 \%)$ & $x^{2}(2)=3.04, p=0.219$ \\
\hline
\end{tabular}

*Significant at $p<0.05$

example, in a sample of 283 adult patients with bipolar I or II disorder, a prior study failed to find differences in clinical outcomes between lithium plus optimized personalized treatment (OPT) and OPT-only (Nierenberg et al. 2013). However, the OPT-only group was more frequently prescribed second-generation antipsychotics (Nierenberg et al. 2013). Similarly, a trial of lithium monotherapy, sertraline monotherapy or combination therapy of lithium plus sertraline in 142 patients with bipolar II disorder, reported no differences in treatment response or switch rate among groups (Altshuler et al. 2017). Consistent with our observation of increased rateof-dropout in the $\mathrm{DVP}+\mathrm{Li}$ arm versus the $\mathrm{DVP}+\mathrm{PBO}$ arm, the study found an increased rate-of-dropout in the combination therapy arm than in the monotherapy arms (Altshuler et al. 2017). An open-label trial of lithium monotherapy, divalproex monotherapy or their combination for bipolar I relapse prevention in 330 participants found that combination therapy with lithium and divalproex was superior to monotherapy with divalproex but not superior to monotherapy with lithium for relapse prevention (Geddes et al. 2011). Taken together with findings from our pilot study, these data suggest an equivocal additional benefit of combination therapies (vs. monotherapy) for bipolar disorders.

Interestingly, while the rate-of-dropout differed between the $\mathrm{DVP}+\mathrm{Li}$ and $\mathrm{DVP}+\mathrm{PBO}$ arms, with a 3.04 times higher risk-of-dropout in the DVP $+\mathrm{Li}$ arm, the rate-of-dropout did not differ significantly between the DVP + QTP and DVP + PBO arms. It is essential to note that this null effect may be due to the limited number of participants per treatment arm and resulting low power. Indeed, an examination of Fig. 3 suggests a potential difference in rate-of-dropout, in the expected direction, between the DVP + QTP and DVP + PBO arms. Moreover, provisional findings from this pilot study did 
Table 3 Reported side effects of at least moderate intensity judged to be possibly, probably, or definitely related to study medication

\begin{tabular}{|c|c|c|c|c|}
\hline & $\begin{array}{l}\text { Entire cohort } \\
N=75\end{array}$ & $\begin{array}{l}\mathrm{DVP}+\mathrm{PBO} \\
\mathrm{n}=24\end{array}$ & $\begin{array}{l}\mathrm{DVP}+\mathrm{Li} \\
\mathrm{n}=25\end{array}$ & $\begin{array}{l}\mathrm{DVP}+\mathrm{QTP} \\
\mathrm{n}=\mathbf{2 6}\end{array}$ \\
\hline Average number of side effects reported & $5.4(2.6)$ & $6.5(2.2)$ & $4.5(2.4)$ & $5.4(3.0)$ \\
\hline Increased appetite & $34(45.3 \%)$ & $11(14.7 \%)$ & $12(16 \%)$ & $11(14.7 \%)$ \\
\hline Sedation & $32(42.7 \%)$ & $9(12 \%)$ & $2(2.7 \%)$ & $21(28 \%)$ \\
\hline Dry mouth & $29(38.7 \%)$ & $6(8 \%)$ & $8(10.7 \%)$ & $15(20 \%)$ \\
\hline Nausea & $25(33.3 \%)$ & $8(10.7 \%)$ & $9(12 \%)$ & $8(10.7 \%)$ \\
\hline Tiredness & $23(30.7 \%)$ & $7(9.3 \%)$ & $4(5.3 \%)$ & $12(16 \%)$ \\
\hline Increased thirst & $21(28 \%)$ & $2(2.7 \%)$ & $7(9.3 \%)$ & $12(16 \%)$ \\
\hline Upset stomach & $21(28 \%)$ & $8(10.7 \%)$ & $7(9.3 \%)$ & $6(8 \%)$ \\
\hline Diarrhea & $20(26.7 \%)$ & $9(12 \%)$ & $7(9.3 \%)$ & $4(5.3 \%)$ \\
\hline Gastrointestinal problems & $18(24 \%)$ & $7(9.3 \%)$ & $8(10.7 \%)$ & $3(4 \%)$ \\
\hline Increased urinary frequency & $16(21.3 \%)$ & $3(4 \%)$ & $9(12 \%)$ & $4(5.3 \%)$ \\
\hline Word finding difficulties & $14(18.7 \%)$ & $1(1.3 \%)$ & $6(8 \%)$ & $7(9.3 \%)$ \\
\hline Slurred speech & $10(13.3 \%)$ & $0(0 \%)$ & $3(4 \%)$ & $7(9.3 \%)$ \\
\hline Ataxia* $^{*}$ & $10(13.3 \%)$ & $0(0 \%)$ & $3(4 \%)$ & $7(9.3 \%)$ \\
\hline Weakness & $9(12 \%)$ & $2(2.7 \%)$ & $2(2.7 \%)$ & $5(6.7 \%)$ \\
\hline Dizzy/lightheaded & $9(12 \%)$ & $0(0 \%)$ & $2(2.7 \%)$ & $7(9.3 \%)$ \\
\hline Edema & $9(12 \%)$ & $3(4 \%)$ & $2(2.7 \%)$ & $4(5.3 \%)$ \\
\hline Impaired memory & $9(12 \%)$ & $1(1.3 \%)$ & $3(4 \%)$ & $5(6.7 \%)$ \\
\hline Feeling dull & $9(12 \%)$ & $0(0 \%)$ & $2(2.7 \%)$ & $7(9.3 \%)$ \\
\hline Cognitive slowing & $9(12 \%)$ & $0(0 \%)$ & $2(2.7 \%)$ & $7(9.3 \%)$ \\
\hline Joint/muscle aches & $9(12 \%)$ & $4(5.3 \%)$ & $2(2.7 \%)$ & $3(4 \%)$ \\
\hline Tactile sensations & $7(9.3 \%)$ & $1(1.3 \%)$ & $1(1.3 \%)$ & $5(6.7 \%)$ \\
\hline Increased appetite & $34(45.3 \%)$ & $11(14.7 \%)$ & $12(16 \%)$ & $11(14.7 \%)$ \\
\hline
\end{tabular}

*Significant at $p<0.05$

Table 4 Longitudinal outcomes in YMRS and HAM-D: monotherapy versus aggregated combination therapies

\begin{tabular}{|c|c|c|c|c|c|c|}
\hline \multirow[t]{2}{*}{ Predictors } & \multicolumn{3}{|l|}{ YMRS } & \multicolumn{3}{|l|}{ HAM-D } \\
\hline & Estimates & $\mathrm{Cl}$ & $p$ & Estimates & $\mathrm{Cl}$ & $p$ \\
\hline Group [combination] & 0.39 & $-2.69-3.47$ & 0.802 & 1.41 & $-1.32-4.15$ & 0.307 \\
\hline Time [days] & -0.13 & -0.17 to -0.08 & $<0.001^{* * *}$ & -0.05 & -0.09 to -0.02 & $0.005^{* *}$ \\
\hline Site [VA] & 0.37 & $-3.34-4.07$ & 0.844 & -4.59 & -8.08 to -1.11 & $0.011^{*}$ \\
\hline Group [combination] $\times$ time & -0.02 & $-0.08-0.04$ & 0.443 & 0.02 & $-0.03-0.07$ & 0.359 \\
\hline
\end{tabular}

Monotherapy: divalproex plus placebo; combination: combination therapy of divalproex plus blinded lithium and divalproex plus blinded quetiapine VA Palo Alto

${ }^{*} p<0.05,{ }^{* *} p<0.01,{ }^{* * *} p<0.001$

Table 5 Longitudinal Outcomes in YMRS and HAM-D: monotherapy versus individual combination therapies

\begin{tabular}{|c|c|c|c|c|c|c|}
\hline \multirow[t]{2}{*}{ Predictors } & \multicolumn{3}{|l|}{ YMRS } & \multicolumn{3}{|l|}{ HAM-D } \\
\hline & Estimates & $\mathrm{Cl}$ & $p$ & Estimates & $\mathrm{Cl}$ & $p$ \\
\hline Group [Li] & 1.80 & $-1.77-5.37$ & 0.319 & 2.29 & $-0.87-5.45$ & 0.153 \\
\hline Group [QTP] & -0.83 & $-4.36-2.69$ & 0.640 & 0.66 & $-2.46-3.78$ & 0.675 \\
\hline Time [days] & -0.13 & -0.18 to -0.08 & $<0.001^{* * *}$ & -0.05 & -0.09 to -0.02 & $0.004^{* *}$ \\
\hline Site $[V A]$ & 0.41 & $-3.31-4.13$ & 0.826 & -4.60 & -8.14 to -1.07 & $0.011^{*}$ \\
\hline Group $[\mathrm{Li}] \times$ time & -0.06 & $-0.14-0.01$ & 0.108 & -0.00 & $-0.06-0.05$ & 0.878 \\
\hline Group [QTP] $\times$ time & 0.00 & $-0.06-0.07$ & 0.919 & 0.04 & $-0.01-0.09$ & 0.124 \\
\hline
\end{tabular}

PBO: monotherapy divalproex plus placebo [reference group]; Li: combination therapy of divalproex plus blinded lithium; QTP: divalproex plus blinded quetiapine; VA: Palo Alto VA

${ }^{*} p<0.05,{ }^{* *} p<0.01,{ }^{* * *} p<0.001$ 


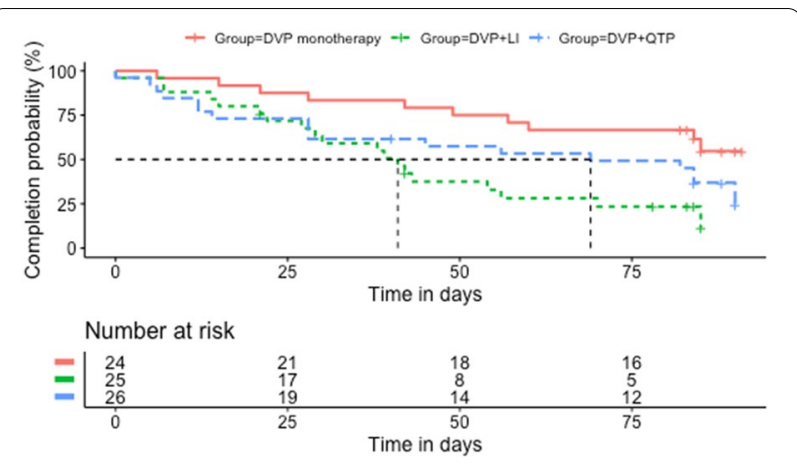

Fig. 2 Kaplan-Meier time-to-event curves

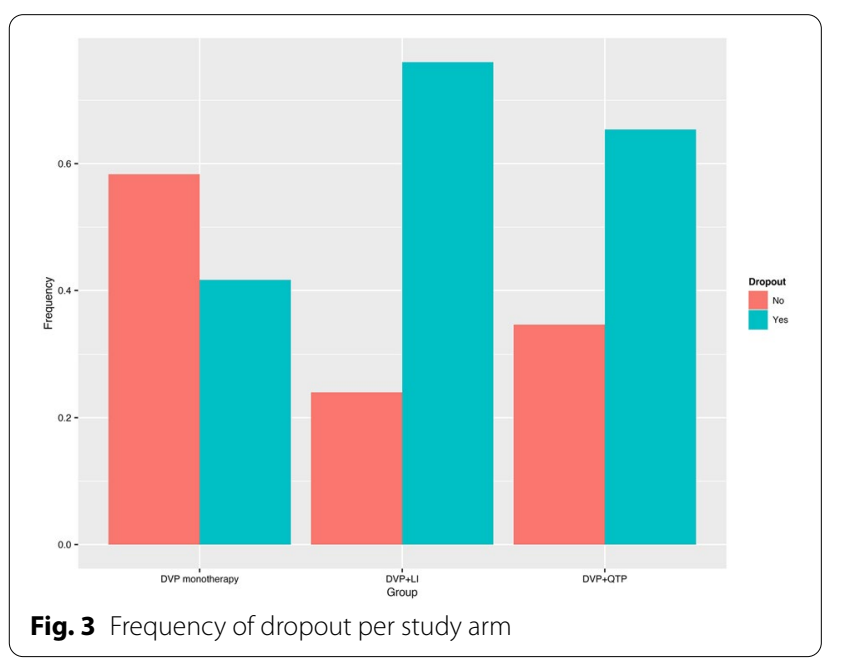

not suggest between-group differences in the effect of side-effect severity and number of side effects on rate-ofdropout, which may also be due to being underpowered. Further studies, with adequate numbers of participants to be fully powered, are needed to elucidate between-group differences in side effect severity on rate-of-dropout.

A consequential limitation of the present pilot study is the small sample size and differential attrition across treatment arms. The higher rate-of-dropout in the $\mathrm{DVP}+\mathrm{Li}$ arm may have also violated the missing at random assumption of linear mixed models and increased Type II error in a potentially significant fashion. Future studies would benefit by the use of larger sample sizes to estimate a pattern mixture model or a joint model of the longitudinal outcome process and the time-to-dropout process, thereby adjusting for the effect of dropout in estimating longitudinal differences in YMRS and HAM-D among the 3 treatment arms. In addition, the generalizability of our results may be tempered by the low to moderate YMRS cutoff score of $\geq 15$. Further, future study designs could consider potential differential response patterns between individuals with syndromal hypomania vs. mania.

\section{Conclusions}

Three provisional implications, cautioned by the small sample size of this pilot study, may be drawn from the present double-blind, placebo-controlled ambulatory pilot trial. First, the preliminary findings suggest no added benefit of combination therapy versus monotherapy with divalproex sodium for manic symptoms in ambulatory subjects with bipolar I disorder; second, divalproex plus blinded lithium could be associated with greater risk of dropout than divalproex alone; and third, study retention in our pilot investigation did not appear to be associated with differences in side effect severity or the number of side effects reported between

Table 6 Cox regression on retention for monotherapy versus individual combination therapies

\begin{tabular}{|c|c|c|c|c|c|c|}
\hline Variable & B & SE & Wald & $P$ & Hazard ratio & $95 \% \mathrm{Cl}$ \\
\hline \multicolumn{7}{|l|}{ Group } \\
\hline PBO & - & - & - & - & - & - \\
\hline $\mathrm{Li}$ & $1.11(1.11)$ & $0.53(0.52)$ & $2.11(2.14)$ & $0.035^{*}(0.033)$ & $3.04(3.04)$ & $1.08-8.57(1.10-8.43)$ \\
\hline QTP & $0.27(0.17)$ & $0.58(0.59)$ & $0.46(0.29)$ & $0.644(0.773)$ & $1.31(1.18)$ & $0.42-4.06(0.38-3.73)$ \\
\hline Side effect & $0.08(0.09)$ & $0.11(0.11)$ & $0.76(0.78)$ & $0.448(.435)$ & 1.09 (1.09) & $0.88-1.35(0.88-1.35)$ \\
\hline Side effect severity $\times \mathrm{Li}$ & $-0.05(-0.04)$ & $0.12(0.13)$ & $-0.43(-0.33)$ & $0.666(0.742)$ & $0.95(0.96)$ & $0.75-1.21(0.75-1.23)$ \\
\hline Side effect severity $\times$ QTP & $-0.03(-0.03)$ & $0.11(0.11)$ & $-0.30(-0.29)$ & $0.763(0.769)$ & $0.97(0.97)$ & $0.77-1.21(0.78-1.21)$ \\
\hline YMRS & $0.00(0.00)$ & $0.03(0.03)$ & $0.11(0.84)$ & $0.914(0.933)$ & $1.00(1.00)$ & $0.95-1.06(0.95-1.06)$ \\
\hline HAM-D & $0.06(0.06)$ & $0.04(0.04)$ & $1.64(1.59)$ & $0.101(0.113)$ & $1.06(1.06)$ & $0.99-1.14(0.99-1.14)$ \\
\hline Site & -0.68 & 1.06 & -0.64 & 0.521 & 0.51 & $0.06-4.06$ \\
\hline
\end{tabular}

Estimates for the model that excluded site are shown in parentheses

PBO: monotherapy divalproex plus placebo; Li: combination therapy of divalproex plus blinded lithium; QTP: divalproex plus blinded quetiapine

"-" indicates reference group

*Significant at $p<0.05$ 
Table 7 Cox regression on retention for monotherapy versus aggregated combination therapies

\begin{tabular}{|c|c|c|c|c|c|c|}
\hline Variable & B & SE & Wald & $P$ & Hazard ratio & $95 \% \mathrm{Cl}$ \\
\hline \multicolumn{7}{|l|}{ Group } \\
\hline Monotherapy & - & - & - & - & - & - \\
\hline Combination therapy & $0.93(0.92)$ & $0.48(0.48)$ & $1.94(1.90)$ & $0.052(0.058)$ & $2.56(2.51)$ & $0.99-6.61(0.97-6.50)$ \\
\hline Side effect severity & $0.02(0.02)$ & $0.08(0.09)$ & $0.27(0.27)$ & $0.785(0.785)$ & $1.02(1.02)$ & $0.87-1.21(0.86-1.21)$ \\
\hline Side effect severity $\times$ group & $0.01(0.00)$ & $0.09(0.09)$ & $-0.43(0.01)$ & $0.939(0.992)$ & $1.01(1.00)$ & $0.85-1.20(0.84-1.19)$ \\
\hline YMRS & $0.00(-0.00)$ & $0.03(0.03)$ & $0.04(-0.03)$ & $0.965(0.973)$ & $1.00(1.00)$ & $0.95-1.06(0.95-1.05)$ \\
\hline HAM-D & $0.06(0.07)$ & $0.03(0.03)$ & $1.74(2.06)$ & $0.082\left(0.039^{*}\right)$ & $1.06(1.07)$ & $0.99-1.14(1.00-1.14)$ \\
\hline Site & -1.02 & 1.05 & -0.97 & 0.334 & 0.36 & $0.05-2.85$ \\
\hline
\end{tabular}

Estimates for the model that excluded site are shown in parentheses

Monotherapy: divalproex plus placebo; combination: combination therapy of divalproex plus blinded lithium and divalproex plus blinded quetiapine

Monotherapy ("-") was used as the reference group

*Significant at $p<0.05$

treatment arms, with attrition and low statistical power critical contributors.

\author{
Abbreviations \\ DVP: Divalproex; PBO: Placebo; Li: Lithium; QTP: Quetiapine; BDI: Bipolar I \\ disorder; SCID: Structured Clinical Interview for DSM-IV; YMRS: Young Mania \\ Rating Scale; HAM-D: Hamilton Rating Scale for Depression; CGI-BP: Clinical \\ Global Impression Scale for Bipolar Disorder; SOFAS: Social and Occupational \\ Functioning Scale.
}

\section{Acknowledgements}

Not applicable.

\section{Authors' contributions}

TS conceived and planned the study. TS, VC and GF carried out the study. IG and SA analyzed the data. VC, TS, MO, SA and IG interpreted the results. Although VC led the writing of the manuscript, all authors contributed to the writing of the manuscript. All authors, with the exception of IG, read and approved the final manuscript.

\section{Funding}

Funding for this study was provided by NIMH R01MH069801. TS and MO are full time employees of the U.S. Department of Veterans Affairs. The supporters had no role in the design, analysis, interpretation, or publication of this study. ClinicalTrials.gov identifier: NCT00183443.

\section{Availability of data and materials}

The datasets used and/or analyzed during the current study are available from the corresponding author on reasonable request.

\section{Declarations}

\section{Ethics approval and consent to participate}

The Institutional Review Boards of the University of Texas Southwestern Medical Center and the Stanford University School of Medicine approved this study. All participants provided written informed consent.

\section{Consent for publication}

Not applicable.

\section{Competing interests}

TS in the past 36 months has reported grants from Pathway Genomics, Stanley Medical Research Institute, Elan Pharma International Limited, Merck and Co., and Sunovion Pharmaceuticals; consulting fees from Allergan, Impel NeuroPharma Inc., Intraceluular Therapies, and Sunovion Pharmaceuticals; honoraria from CME Institute, Health and Wellness Partners Inc., and CMEology; royalties from Wolters Kluwer Health (UpToDate), Jones and Bartlett, American Psychiatric Association Press, and Hogrefe Publishing. MO in the past 36 months has reported consulting fees from Johnson \& Johnson, Sage Therapeutics, and Alkermes; and grant support from Palo Alto Health Sciences, Inc. VC in the past 36 months has reported grants from the National Cancer Institute and royalties from UpToDate. SA, IG, and GF declare that they have no competing interests.

\section{Author details}

${ }^{1}$ Bipolar and Depression Research Program, VA Palo Alto Health Care System, 3801 Miranda Avenue (151T), Palo Alto, CA 94304, USA. ${ }^{2}$ Department of Psychiatry and Behavioral Sciences, Stanford University School of Medicine, 401 Quarry Road, Stanford, CA 94305, USA.

Received: 27 July 2021 Accepted: 18 January 2022

Published online: 02 March 2022

\section{References}

Altshuler LL, Sugar CA, McElroy SL, et al. Switch rates during acute treatment for bipolar II depression with lithium, sertraline, or the two combined: a randomized double-blind comparison. Am J Psychiatry. 2017;174(3):266-76.

Brooks JO, Goldberg JF, Ketter TA, et al. Safety and tolerability associated with second-generation antipsychotic polytherapy in bipolar disorder: findings from the systematic treatment enhancement program for bipolar disorder. J Clin Psychiatry. 2010;72(2):240-7. https://doi.org/10.4088/jcp. 09m05214yel.

Efron B. Forcing a sequential experiment to be balanced. Biometrika. 1971;58(3):403-17. https://doi.org/10.1093/biomet/58.3.403.

Faedda GL, Tondo L, Baldessarini RJ, Suppes T, Tohen M. Outcome after rapid vs gradual discontinuation of lithium treatment in bipolar disorders. Arch Gen Psychiatry. 1993;50(6):448-55.

First M, Gibbon M, Spitzer R, et al. Structured clinical interview for DSM-IV axis I disorders-research version (SCID-I). New York: Biometrics Research Department, New York State Psychiatric Institute; 1996.

Gaudiano BA, Weinstock LM, Miller IW. Improving treatment adherence in bipolar disorder: a review of current psychosocial treatment efficacy and recommendations for future treatment development. Behav Modif. 2008;32(3):267-301.

Geddes JR, Collaborators TBIA, The BALANCE investigators and collaborators, et al. Lithium plus valproate combination therapy versus monotherapy for relapse prevention in bipolar I disorder (BALANCE): a randomised open-label trial. FOCUS. 2011;9(4):488-99. https://doi.org/10.1176/foc.9. 4.foc 488 . 
Grande I, Vieta E. Pharmacotherapy of acute mania: monotherapy or combination therapy with mood stabilizers and antipsychotics? CNS Drugs. 2015;29(3):221-7.

Hamilton M. A rating scale for depression. J Neurol Neurosurg Psychiatry. 1960;23:56-62.

Hamilton M. Development of a rating scale for primary depressive illness. Br J Soc Clin Psychol. 1967:6:278-96.

Kassambara MK. survminer: drawing survival curves using 'ggplot2'. R package version 0 4. 2018;3.

Lüdecke D. sjPlot: data visualization for statistics in social science. R package version. 2018;2(1).

Nierenberg AA, Friedman ES, Bowden CL, Sylvia LG, Thase ME, Ketter T, Ostacher MJ, Leon AC, Reilly-Harrington N, losifescu DV, Pencina M. Lithium treatment moderate-dose use study (LiTMUS) for bipolar disorder: a randomized comparative effectiveness trial of optimized personalized treatment with and without lithium. Am J Psychiatry. 2013;170(1):102-10,

Pinheiro J, Bates D, DebRoy S, Sarkar D, Team RC, et al. nlme: linear and nonlinear mixed effects models. R package version. 2013;3(1):111.

Raudenbush SW, Bryk AS. Hierarchical linear models: applications and data analysis methods. Thousand Oaks: SAGE; 2002.

Scott J. Using Health Belief Models to understand the efficacy-effectiveness gap for mood stabilizer treatments. Neuropsychobiology. 2002;46(Suppl 1):13-5.

Snijders TA, Bosker RJ. Multilevel analysis: an introduction to basic and advanced multilevel modeling. Los Angeles: SAGE; 1999. https://doi.org/ 10.1111/j.2041-210x.2012.00261.x.

Suppes T, Baldessarini RJ, Faedda GL, Tohen M. Risk of recurrence following discontinuation of lithium treatment in bipolar disorder. Arch Gen Psychiatry. 1991;48(12):1082-8.

Team R. RStudio: integrated development for R. RStudio. 2020.

Therneau T. A package for survival analysis in R. R package version 3.2-3. 2020. 2020.

Wickham H. Tidyverse: easily install and load the "Tidyverse". R package version 1.2.1. Vienna: R Core Team. 2017.

Williams J. A structured interview guide for the Hamilton depression rating scale. Arch Gen Psychiatry. 1988;45(8):742.

Winder C, Dowlatabadi Z, Miller-Zarneke T. The core team. In: Producing animation. Boca Raton: CRC Press; 2019. p. 59-83. https://doi.org/10.1201/ 9780429490521-4.

Yatham LN, Kennedy SH, Parikh SV, et al. Canadian network for mood and anxiety treatments (CANMAT) and international society for bipolar disorders (ISBD) 2018 guidelines for the management of patients with bipolar disorder. Bipolar Disord. 2018;20(2):97-170. https://doi.org/10.1111/bdi. 12609.

Young R, Biggs J, Ziegler V, Meyer D. A rating scale for mania: reliability, validity and sensitivity. Br J Psychiatry. 1978;133(5):429-35.

\section{Publisher's Note}

Springer Nature remains neutral with regard to jurisdictional claims in published maps and institutional affiliations.

\section{Submit your manuscript to a SpringerOpen ${ }^{\circ}$ journal and benefit from:}

- Convenient online submission

- Rigorous peer review

- Open access: articles freely available online

- High visibility within the field

- Retaining the copyright to your article 